\title{
A representação subjacente dos glides e suas consequências para a análise do glide em Português brasileiro
}

\author{
The underlying representation of glides and its \\ consequences to the analysis of glide in Brazilian \\ Portuguese
}

La representación subyacente de los glides y sus consecuencias para el análisis del glide en el Portugués brasileño

\author{
iD (9) Taíse Simioni \\ Universidade Federal do Pampa (UNIPAMPA), Bagé, Brasil. \\ E-mail: taisesimioni@unipampa.edu.br
}

Resumo: Neste trabalho temos por objetivo retomar a discussão de Levi (2004) sobre a possibilidade de existência de glides subjacentes e, a partir disso, discutir se o glide subjacente ocorre em português brasileiro (PB). Levando em consideração a parcial predizibilidade do glide e seu comportamento no processo de palatalização e na alomorfia de diminutivo, chegamos à conclusão de que há somente glides derivados em PB. Isso nos leva a incluir esta língua, na tipologia de Levi (2004), como aquela em que, na subjacência, só há vogais, que podem se realizar como vogais ou glides. Dentro deste tipo, o PB seria classificado como uma língua "normal".

Palavras-chave: Glide. Representação subjacente. Português brasileiro. 
Abstract: In this paper, we aim to resume the discussion by Levi (2004) about the undelying glides and, from this, to discuss whether the underlying glide occurs in Brazilian Portuguese (BP). Starting from the partial predictability of the occurence of the glide, and the behavior of this segment in the process of palatization and in the alomorphy of diminutive, we reached the conclusion that there are only derived glides in BP, and this leads us to include this language, according to the tipology proposed by Levi (2004), as that in which, subjacently, there are only vowels, that can be realized as vowels or glides as well. Within this type, the BP would be classified as a "normal" language.

Keywords: Glide. Underlying representation. Brazilian Portuguese.

Resumen: En este artículo, nuestro objetivo es reanudar la discusión de Levi (2004) sobre la posibilidad de glides subyacentes y, a partir de esto, discutir si el glide subyacente se produce en el portugués brasileño (PB). Teniendo en cuenta la predictibilidad parcial del glide y su comportamiento en el proceso de palatalización y en el alomorfía del diminuto, llegamos a la conclusión de que solamente existen glides derivados en el PB. Esto nos lleva a incluir esta lengua, en la tipología de Levi (2004), como la que, en el nivel subyacente, solamente presenta vocales, que se pueden realizar como vocales o glides. Dentro de este tipo, el PB podría ser clasificado como una lengua "normal".

Palabras clave: Glide. Representación subyacente. Portugués brasileño.

Submetido em 26 de julho de 2019.

Aceito em 24 de outubro de 2019.

Publicado em 06 de fevereiro de 2020. 


\section{Introdução}

Como afirma Hyman (2003 [1985]), "talvez o tipo de segmento mais problemático para todas as teorias fonológicas seja a classe dos glides" (2003, p. 77). Isso ocorre, principalmente, porque eles apresentam características tanto de vogais quanto de consoantes. Levi (2004), na contramão de muitas análises segundo as quais glides subjacentes não existem, propõe-se a defender tal existência. Para a autora, "glides derivados seguem o padrão das vogais e possuem a mesma representação delas em termos de traços. Glides subjacentes, por outro lado, podem ser agrupados com as consoantes e formam uma classe natural com elas" (LEVI, 2004, p. 2). Segundo Levi, a comprovação de que glides subjacentes existem não tem por consequência a suposição de que eles existam em todas as línguas do mundo. Nosso objetivo, então, neste trabalho, é retomar a discussão de Levi (2004) sobre a possibilidade de existência de glides subjacentes e, a partir disso, discutir se o glide subjacente ocorre em português brasileiro (PB). A retomada de Levi (2004), feita na segunda seção deste trabalho, implica a exposição de diferentes representações possíveis para o glide subjacente, como mostra a autora. A partir dessa discussão será possível verificar a que tipo de língua, de acordo com a tipologia proposta por Levi, pertence o PB.

Com relação à representação do glide subjacente, Levi discute quatro possibilidades: marcação lexical, uso do traço [ \pm consonantal], Vowel-Place Theory e Revised Articulator Theory. Essa discussão leva à conclusão de que glides subjacentes, por um lado, e vogais e glides derivados, por outro, distinguem-se através de traços, como vários processos fonológicos podem atestar. Tendo isto em mente, na terceira seção deste trabalho discutimos sobre a falta de evidências para a existência de glides subjacentes em PB, o que está de acordo com estudos clássicos sobre o PB, como os de Lopez (1979) e Bisol (1989, 1994, 1999). A diferença, entretanto, reside no fato de que tais estudos partem do pressuposto de que não existem glides subjacentes nas línguas do mundo. O presente 
A representação subjacente dos glides e suas consequências...

Taíse Simioni

trabalho, apoiado em Levi (2004), admite a existência de tais segmentos. Neste sentido, é necessário contrapor o comportamento de um glide derivado com o que poderia ser um glide subjacente em PB. Para tal, foram analisadas a predizibilidade parcial da realização das vogais altas, a palatalização de [t] e [d] e a alternância entre os morfemas de diminutivo -inho e -zinho. A conclusão é a de que não existem glides subjacentes em $\mathrm{PB}$, uma vez que não são encontrados, nesta língua, processos que atestem a diferença entre vogais e glides subjacentes em termos de traços. Na quarta seção, apresentamos a tipologia proposta por Levi em relação à presença ou ausência de glides subjacentes nas línguas do mundo. De acordo com tal tipologia, o PB pertenceria ao tipo III, uma língua em que só há vogais subjacentemente, mas em que tais vogais podem se realizar como glides. Ainda no interior desta seção, apresentamos a distinção que Levi faz entre línguas "normais" e "vocálicas". Ambas não apresentam glides subjacentes, mas, no segundo tipo, há a presença de vogais em contextos nos quais a superficialização de um glide seria esperado em função dos padrões da língua.

\section{A representação dos glides subjacentes}

Como mencionamos na introdução, Levi (2004), na tese em que defende a existência de glides subjacentes, discute quatro propostas de análise para os glides subjacentes: marcação lexical, uso do traço [ \pm consonantal] e duas alternativas referentes à geometria de traços (Vowel-Place Theory e Revised Articulator Theory). A autora ressalta que, embora haja outras possibilidades, selecionou essas quatro por serem as mais difundidas na literatura sobre o assunto. 
A representação subjacente dos glides e suas consequências...

\subsection{Marcação lexical}

De acordo com a marcação lexical, glides são subjacentemente marcados como segmentos que não podem constituir núcleo de sílaba. Para esta proposta, essa é a única diferença entre glides e vogais; os dois tipos de segmentos são, portanto, iguais em termos de traços (a menos que a diferença seja marcada pelo traço [silábico]; neste caso, haverá essa distinção em termos de traços). Isso significa que, nesta proposta, processos que fazem menção a traços não podem fazer distinção entre vogais e glides subjacentes. Os únicos processos capazes de realizar tal distinção são aqueles que se referem a posições silábicas.

Levi (2004) afirma que essa proposta pode ser representada por análises segundo as quais há uma camada CV entre a camada da sílaba e a camada melódica, como aquela de Clements e Keyser (1983), para os quais a diferença entre glides e vogais reside na sua afiliação à camada CV: vogais afiliam-se a V, enquanto glides, a C. A afiliação de glides a uma posição C os impede de ocuparem o núcleo de uma sílaba.

Segundo Levi (2004), essa possibilidade de análise pode ser considerada o oposto da proposta de Levin (1985), segundo a qual há vogais lexicalmente marcadas para serem núcleos de sílaba. De fato, Levin rejeita a hipótese de que haja segmentos lexicalmente marcados para não constituírem núcleo de sílaba (o que equivale a cabeça silábico): "cabeças podem ser marcados nas representações lexicais subjacentes, enquanto a propriedade de não ser cabeça não pode" (LEVIN, 1985, p. 2).

\subsection{Uso do traço [ \pm consonantal]}

Conforme esta proposta, vogais subjacentes são [-consonantais] e glides subjacentes são [+consonantais] (cf., por exemplo, ROSENTHALL, 1994; HALL, 2017). Hayes (1989) também utiliza o traço [consonantal] para diferenciar vogais de glides, no caso de 
A representação subjacente dos glides e suas consequências...

sequências como [yi] e [wu]. Além disso, Zec (2007), em nota, adota esse traço para distinguir vogais de glides subjacentes. Waksler (1990) defende a ideia de que vogais que não alternam com glides são [-consonantais], glides subjacentes são [+consonantais] e glides que alternam com vogais são [0consonantais]. Em uma direção oposta, Hume (1995) e Hume e Odden (1996) defendem a ideia de que o traço [consonantal] deveria ser eliminado. Para tal, trazem uma série de argumentos, que não serão discutidos aqui.

Segundo Levi (2004), por essa proposta de uso do traço [consonantal], os outros traços são os mesmos para os dois tipos de segmentos. Conforme a autora, nesta proposta, quando uma vogal subjacente é realizada como um glide derivado, o traço [-consonantal] é mantido, e a distinção entre a vogal e o glide derivado é feita por suas respectivas posições silábicas: núcleo e não-núcleo, respectivamente.

O uso do traço [consonantal] para diferenciar vogais subjacentes de glides subjacentes tem como consequência o fato de que somente processos que fazem menção a esse traço podem atestar essa distinção. Processos que se referem à sílaba não são capazes de evidenciar a distinção entre vogais (subjacentes ou superficiais) e glides (subjacentes ou superficiais). Na superfície, portanto, glides subjacentes e derivados devem ser tratados da mesma maneira por processos que se referem à sílaba.

Neste momento, é importante destacar que a marcação lexical e o uso do traço [consonantal] são capazes de diferenciar glides subjacentes e vogais em situações diferentes. Para exemplificar isto, tomemos um processo da língua karuk, analisado por Levi (2004) e exemplificado em (1). Trata-se da nasalização de soantes, processo a partir do qual os segmentos $/ \mathrm{r} / \mathrm{e} / \mathrm{w} /$ realizam-se como nasais diante de outra consoante. Glides derivados ([w]) não são atingidos por este processo. 
A representação subjacente dos glides e suas consequências...

/asiw/ $\rightarrow[$ ?ásiw $]$

[?asím-tfak]

/ikriu/ $\rightarrow$ [ikriw]

[ikríw- t]ak] 'dormir'

*[?asíw-t]ak $]$

'fechar os olhos de alguém'

'sentar'

*[ikrím- tfak] 'sentar no caminho'

De acordo com marcação lexical, a diferença entre as raízes de 'dormir' e 'sentar' é o fato de que o último segmento da raiz de 'dormir' seria marcado como não podendo constituir núcleo de sílaba. Já que o glide derivado de [ikriw] e o glide subjacente de [?ásiw] não são distintos em termos de traços, ambos seriam afetados pelo processo de nasalização, o que geraria a forma incorreta *[ikrím-tjak].

O uso do traço [consonantal], diferentemente, é capaz de dar conta deste processo. Em termos de regras, a nasalização poderia ser formalizada de maneira que somente segmentos [+consonantais] fossem atingidos. Como, nesta perspectiva, glides subjacentes são [+consonantais], ao contrário de glides derivados, que são [-consonantais], estaria explicado por que somente os primeiros são afetados pela regra. É importante destacar que, nesta perspectiva, o fato de os glides subjacentes serem considerados [+consonantais] apenas indica que eles se comportam, em relação à aplicação de regras, como consoantes, não necessariamente que eles apresentam diferenças articulatórias ou acústicas em comparação com os glides derivados.

Cabe mencionar aqui as propostas de Nevins e Chitoran (2008) e Padgett (2008), segundo as quais o traço responsável pela diferença entre glides subjacentes e vogais não seria o [consonantal], uma vez que ambos os segmentos seriam [-consonantais], o que os diferenciaria de verdadeiras consoantes, mas o [vocálico]: glides subjacentes seriam segmentos [-vocálicos], e vogais, [+vocálicos]. Para um exemplo de análise que adota essa proposta, sugerimos a leitura de Huang (2014). 
A representação subjacente dos glides e suas consequências...

\subsection{Vowel-Place Theory (VPT)}

De acordo com esta teoria, inserida no contexto da geometria de traços, consoantes e vogais encontram-se separadas em camadas diferentes (CLEMENTS, 1991; CLEMENTS; HUME, 1995). Para Clements e Hume (1995, p. 292), os segmentos podem ser organizados (universalmente); consoantes e vogais são caracterizadas pelos mesmos traços (com o acréscimo do nó de abertura no caso das vogais).

Para que um processo fonológico possa ser considerado natural, nessa proposta, duas situações são possíveis: ou ele afeta traços individuais (os traços terminais, como [contínuo], [labial], etc.) ou ele afeta nós de classe (como o nó laríngeo ou o nó da cavidade oral). Um processo genérico de assimilação entre vogais, como aquele formalizado em (2), é possível porque a consoante interveniente não possui o nó vocálico.

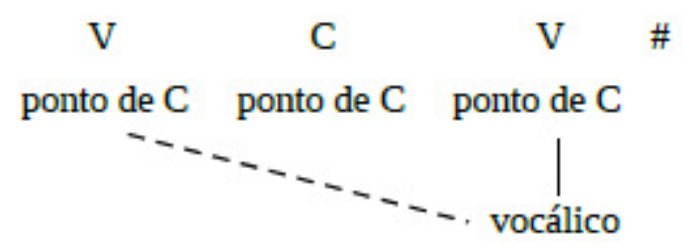

A partir desses pressupostos, Levi (2004) afirma que glides subjacentes podem ser caracterizados pela ausência do nó vocálico, diferenciando-se, desta forma, de vogais e de glides derivados (cf., por exemplo, HUME, 1995), conforme as representações em (3) e (4). Destacamos que a representação de /u/ e /w/ em Levi (2004, p. 32) é um pouco diferente. Nessas representações, o ponto de $\mathrm{C}$ de $/ \mathrm{u} /$ e de $/ \mathrm{w} /$ aparece como [dorsal] e [labial], respectivamente. Isso é claramente um problema de revisão, como pode ser depreendido, diretamente, das representações apresentadas na página 90 de seu texto. 
A representação subjacente dos glides e suas consequências...

Taíse Simioni

(3)

Vogal subjacente: /i/ (e output [j])

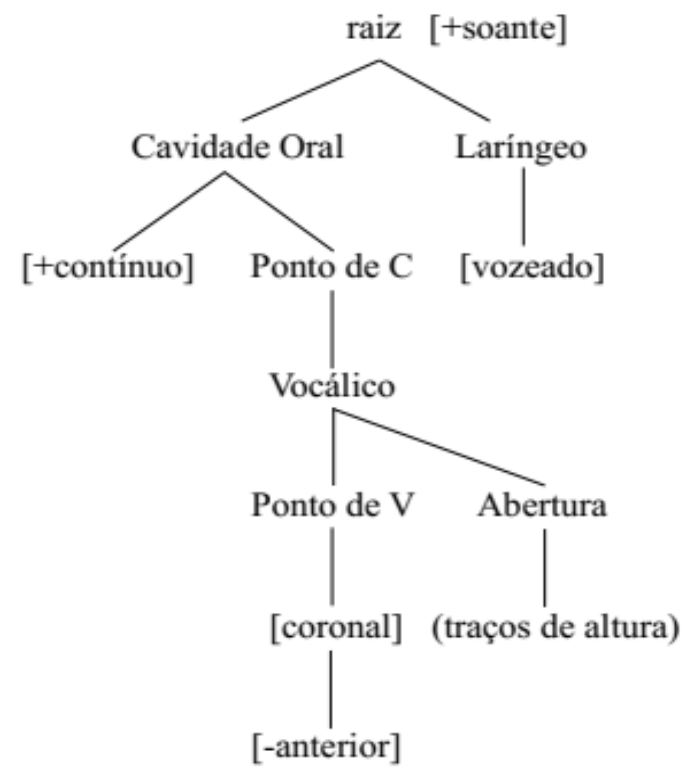

Vogal subjacente: /u/ (e output [w])

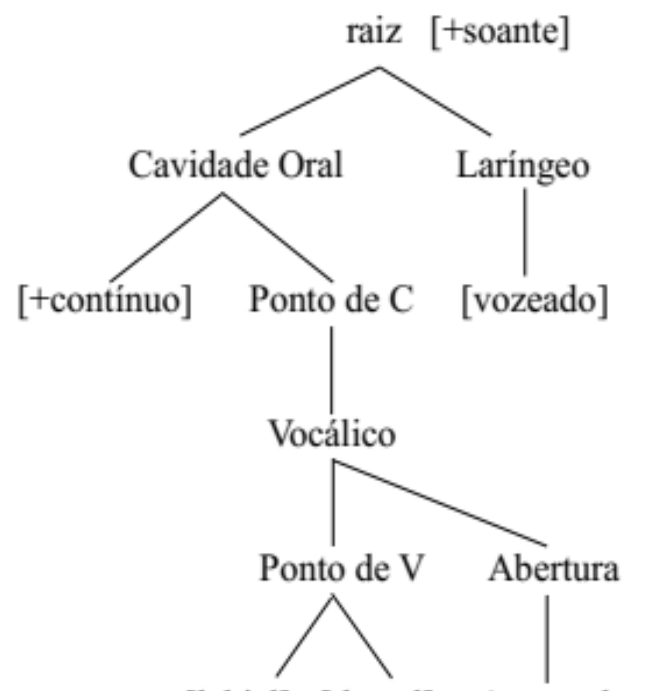

[labial] [dorsal] (traços de altura)

Fonte: Levi (2004, p. 32)

(4)

Glide subjacente: /j/

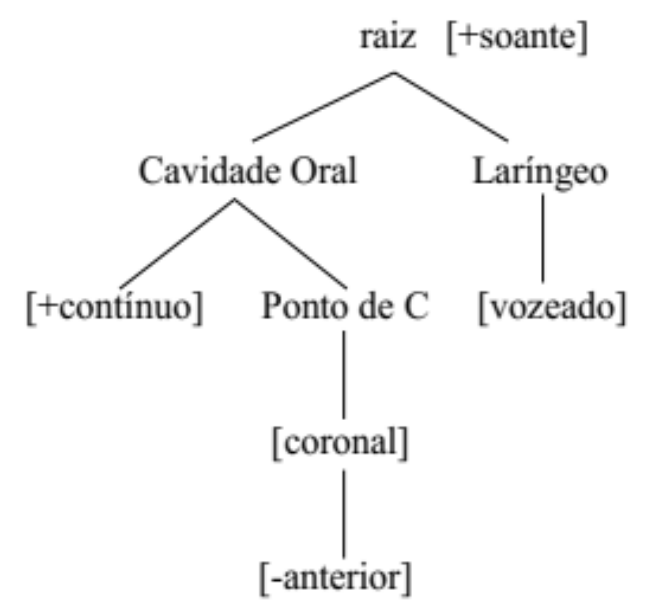

Glide subjacente: /W/

raiz [+soante]

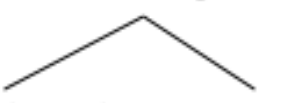

Cavidade Oral

Laríngeo

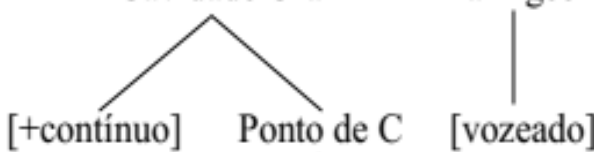<smiles>C1CC1</smiles>

[labial] [dorsal]

Fonte: Levi (2004, p. 32)

VPT prediz que glides subjacentes não bloquearão processos que envolvem traços do ponto de V. Por outro lado, bloquearão processos que se referem a traços do ponto de $C$. 
A representação subjacente dos glides e suas consequências...

\subsection{Revised Articulator Theory (RAT)}

Assim como VPT, RAT é uma teoria inserida no contexto da geometria de traços. Diferentemente da primeira, RAT não propõe camadas diferentes para consoantes e vogais. Para Halle et al. (2000),

à medida que os mesmos articuladores anatômicos estão envolvidos na produção tanto de vogais quanto de consoantes [segundo VPT], a introdução de dois nós de ponto - ponto de $V$ e ponto de $\mathrm{C}$ - trata aspectos fonológicos dos sons da fala (neste caso, os nós geométricos de traços dominando o ponto de articulação) como distintos de seus aspectos articulatórios (neste caso, o ponto de articulação). Na ausência de evidências fortes do contrário, preferimos que uma teoria de geometria de traços mantenha um mapeamento o mais próximo quanto possível entre as propriedades fonéticas e fonológicas da fala, e VPT falha em providenciar tais evidências fortes (HALLE et al., 2000, p. 412).

Todos os segmentos são representados como em (5), conforme proposta de Halle et al. (2000, p. 389). De acordo com o que os autores explicam, esta representação, como qualquer representação de Articulator Theories, baseia-se em características anatômicas do trato vocal. Desta forma, segundo Halle et al. (2000), "cada traço serve como uma instrução para uma ação específica de uma das seis partes móveis do trato vocal: lábios, lâmina da língua, corpo da língua, raiz da língua, palato mole e laringe" (HALLE et al., 2000, p. 388). Essas seis partes são os articuladores. Dependentes deles, na estrutura em (5), estão os traços presos a um articulador (articulator-bound features), que são aqueles executados por apenas um articulador e sempre pelo mesmo articulador, como os traços [arredondado] e [posterior]. Os traços livres de um articulador (articulator-free features), como [contínuo] e [consonantal], diferentemente, são executados por diferentes articuladores em diferentes fonemas. Como explicam Halle et al. (2000), "o articulador que 
A representação subjacente dos glides e suas consequências...

Taíse Simioni

executa o(s) traço(s) livres(s) de um articulador pertencentes a um fonema é chamado de seu articulador designado (designated articulator)" (HALLE et al., 2000, p. 388). Conforme Halle et al. (2000, p. 392), cada articulador contém um traço de articulador (articulator feature); há, portanto, seis traços de articuladores, que são [labial], [coronal], [dorsal], [rinal], [radical] e [glotal]. Estes traços são unários, enquanto os demais são binários.

(5)

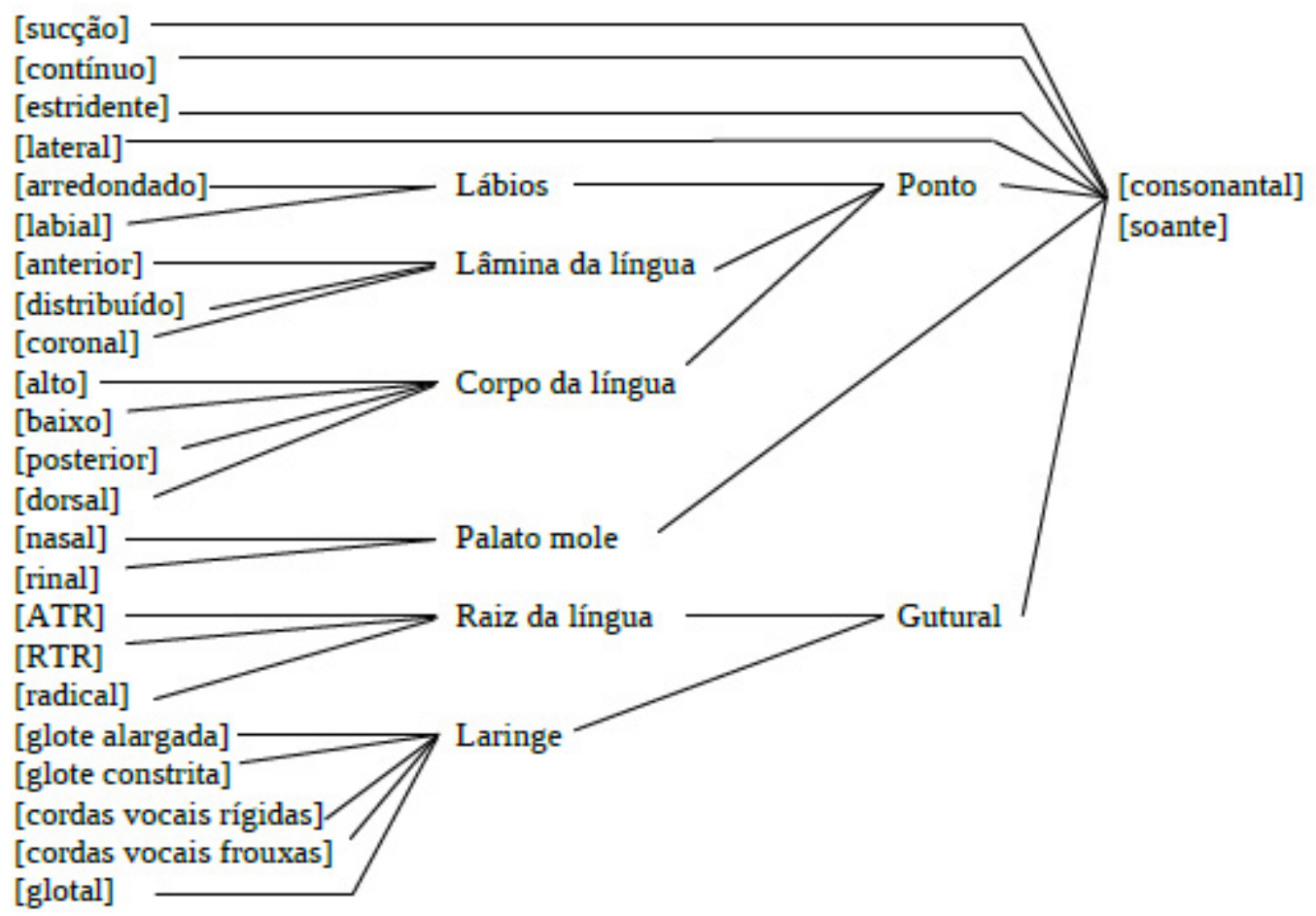

Fonte: Halle et al. (2000, p. 389)

Halle et al. (2000) adotam a proposta de especificação plena de Calabrese (1995). Segundo tal proposta, todos os segmentos são plenamente especificados na representação subjacente, mas nem todos os traços são visíveis para todos os processos.

Além de RAT rejeitar a subespecificação de traços e não propor camadas diferentes para consoantes e vogais, outra diferença em relação a VPT é o fato de que, para Halle et al., “o espraiamento é implementado como operações nos nós terminais da árvore de traços" (HALLE et al., 2000, p. 388). Isto significa que o espraiamen- 
A representação subjacente dos glides e suas consequências...

Taíse Simioni

to parcial, aquele em que traços não dominados exaustivamente por um nó podem espraiar, é permitido.

Conforme Levi (2004), para a RAT, vogais possuem [dorsal] como seu traço de articulador, diferentemente de glides subjacentes, para os quais os traços de articulador são [coronal] e [labial] para /j/ e /W/, respectivamente. Glides derivados apresentam os mesmos traços das vogais que lhes deram origem, o que significa que apresentam [dorsal] como seu traço de articulador. Cabe mencionar que Jaggers (2018) não encontra evidências acústicas para uma proposta segundo a qual [j] seria mais anterior do que [i] em pares análogos em inglês. Isso, segundo o autor, poderia ser um argumento contrário à RAT como explicação para as diferenças entre glides subjacentes e glides derivados. Entretanto, como Jaggers ressalta, faz-se necessário analisar a caracterização acústica desses segmentos em línguas sobre as quais há mais embasamento fonológico para se estabelecer um glide subjacente, porque, nestas línguas, é possível que as pistas acústicas tenham um comportamento diferenciado.

As diferenças representacionais entre glides subjacentes e vogais mostradas por Levi (2004) estão em (6), (7), (8) e (9). Como alerta a autora, nas representações abaixo os traços irrelevantes foram omitidos. É importante mencionar que Halle et al. (2000) não trazem essas representações. Entretanto, Halle (1995, p. 13), embora também não as apresente, afirma que vogais apresentam [dorsal] como seu articulador designado, enquanto os glides [w] e [j] apresentam, respectivamente, [labial] e [coronal] nesta função. 
A representação subjacente dos glides e suas consequências...

Taíse Simioni

/i/ (e [j] derivado) raiz [-consonantal]

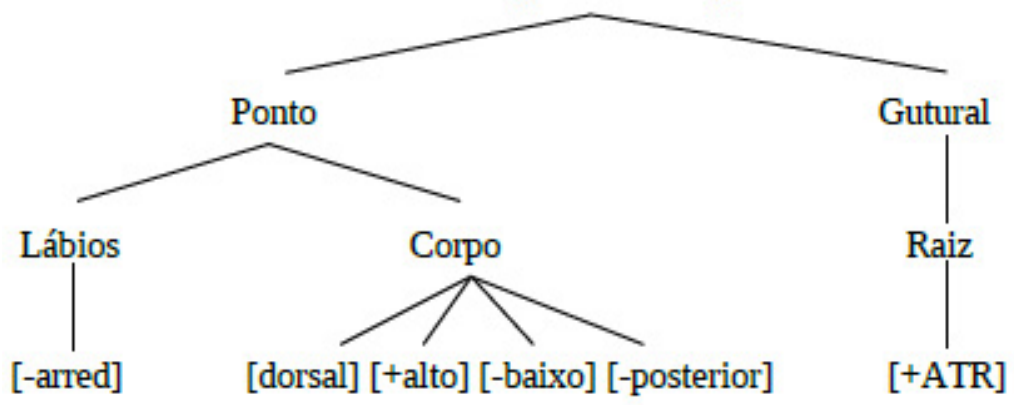

Fonte: Levi (2004, p. 41)

(7)

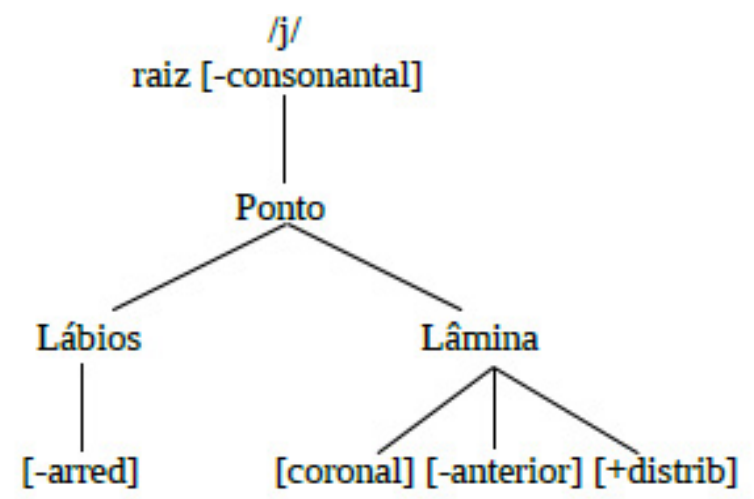

Fonte: Levi (2004, p. 41)

(8)

$/ \mathrm{u} /(\mathrm{e}[\mathrm{w}]$ derivado)

raiz [-consonantal]

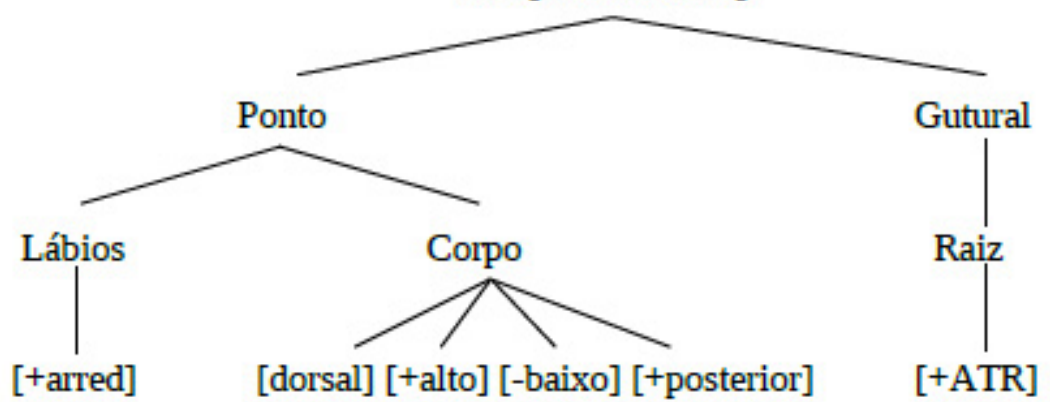

Fonte: Levi (2004, p. 42) 
A representação subjacente dos glides e suas consequências...

(9)

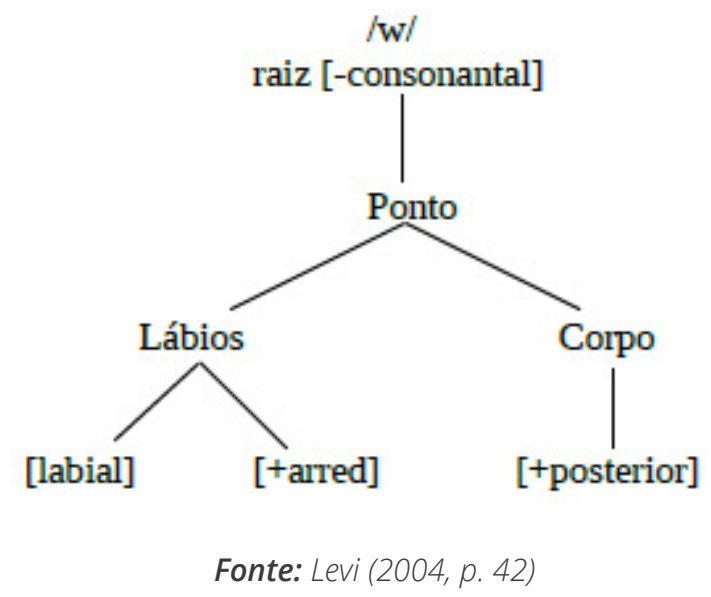

Em função de /j/, /w/ e vogais possuírem diferentes especificações de traços, processos que fazem referência a esses traços podem ser evidência para a distinção entre os segmentos.

Levi (2004) alerta para o fato de que as quatro possibilidades de representação apresentam falhas. Nenhuma delas é capaz de explicar todos os processos discutidos pela autora. Algumas dão conta de determinados processos, enquanto outras dão conta de outros. O que não é surpreendente, segundo a autora, uma vez que nenhuma delas foi desenhada com o objetivo de diferenciar glides subjacentes de vogais. Levi (2004, p. 44-45) afirma, por exemplo, que não há clareza sobre a motivação para que /j/ seja coronal, enquanto /i/ é dorsal.

Apesar de todas as representações apresentarem falhas, ao final de sua análise, Levi (2004) conclui que RAT apresenta uma significativa superioridade em relação à marcação lexical e ao uso do traço [ \pm consonantal], tendo em vista que, em muitos dos processos discutidos pela autora, em diversas línguas, glides subjacentes e glides derivados apresentam comportamentos diferentes que só podem ser explicados a partir da sua constituição em termos de traços, que não o traço [consonantal]. Segundo Levi, RAT apresenta-se como uma análise mais adequada do que VPT, embora ambas as representações consigam dar conta da maioria dos fenômenos apresentados pela autora. É em função de que, 
A representação subjacente dos glides e suas consequências...

Taíse Simioni

segundo Levi (2004), RAT e VPT explicam a maioria dos fenômenos analisados pela autora, diferentemente do que acontece com marcação lexical e uso do traço [ \pm consonantal], que, na próxima seção, nos restringiremos a essas duas propostas. Na sequência, apresentaremos um exemplo em que RAT mostra-se superior em relação à VPT.

O pulaar, dialeto do fula (língua da famíla Níger-Congo), apresenta um processo de gradação consonantal. Nesse dialeto, segundo Levi, as consoantes dos substantivos podem ter alternância em grau (grade) na consoante inicial. O grau da consoante inicial é determinado pelo sufixo marcador de classe. Conforme Levi (2004, p. 77), marcadores de efeito 1 tornam a consoante inicial [-contínua]; marcadores de efeito 2 provocam a pré-nasalização da consoante inicial, se esta for uma oclusiva vozeada; e marcadores de efeito 0 não provocam alterações na consoante inicial (a semântica de tais sufixos marcadores de classe não fica clara). Em (10) transcreveremos apenas os dados relevantes para a discussão sobre a diferença entre glides subjacentes e derivados (LEVI, 2004, p. 77-78). Conforme Levi (2004, p. 74), a seleção que um radical faz de seu marcador é arbitrária. Há uma subcategorização entre o radical e um marcador de classe. 
A representação subjacente dos glides e suas consequências...

Taíse Simioni

(10)

\section{Efeito 0}

a. $\mathrm{w} \sim \mathrm{b}$

wว\}- $\varepsilon \mathrm{r} \varepsilon$

wukk-uru

wibj-5

wil-d $\varepsilon$

waad- $\varepsilon \mathrm{r} \varepsilon$

b. $w \sim g$

wor- $6 \varepsilon$

wur-0

woot-uru

wuuf-re

c. $\mathrm{j} \sim \mathrm{f}$

jeb-re

jim-r $\varepsilon$

jubb-s

jamir-oว-r $\varepsilon$

d. j g

jiit- $\varepsilon \mathrm{r} \varepsilon$

jert- $\varepsilon \mathrm{r} \varepsilon$

jid-be

jecnaa- ${ }^{\mathrm{n}} \mathrm{d} \varepsilon$
Efeito 1

b०ᄀ- $\varepsilon$

bukk-i

bibj-el

bil- $\varepsilon$

baad- $\varepsilon$

gor-k-0

gur- $\varepsilon$

gott-um

guuf- $\varepsilon$

jeb-el

fim-el

jubb- $\varepsilon$

famir- วอ-\}- $\varepsilon$

giit- $\varepsilon$

gert- $\varepsilon$

gid-o

gecnaa-le
Glosa

mboy-on

mbukk-on

'lebre'

'pompom'

'asa'

'armadilha'

'gota (de chuva)'

"gor-on

"gur-on

"gur-on

$\mathrm{n}^{3} \varepsilon 6-0 n$

$n_{\text {fim-on }}$

"giit-on

"gert-on

'olho'

'amendoim'

'amigo'

'túmulo'

Fonte: Levi (2004, p. 77-78)

Segundo Levi, o que esses dados possuem de interessante para a discussão sobre a existência de glides subjacentes é o fato de que [w] ora alterna com [b], ora com [g]. Da mesma maneira, [j] ora alterna com [f], ora com [g]. Segundo a autora, essa alternância é facilmente explicada por RAT, uma vez que, por esta representação, /w/ e /j/ possuem [labial] e [coronal] como articuladores designados, respectivamente, como mostram as representações (9) e (7). Assim, há evidências de que os radicais em (10a) e (10c) apresentem glides subjacentes, porque /w/ alterna com um seg- 
A representação subjacente dos glides e suas consequências...

mento labial - [b] - e /j/ alterna com um segmento coronal - [f]. Com relação aos glides que alternam com [g], é importante observar que eles só ocorrem quando a vogal seguinte é semelhante em termos de anterioridade/posterioridade e arredondamento, como pode ser visto em (10b) e (10d), em que [w] ocorre com [u, o, o] e [j] ocorre com [i, e, ع]. Esta restrição não é observada em (10a) e (10c). Isto leva Levi a concordar com análises anteriores, segundo as quais há um slot vazio antecedendo as vogais em (10b) e (10d). Para preencher esse slot, há, segundo a autora, um espraiamento da vogal inicial do radical. Como prediz RAT, esse slot será preenchido por um segmento dorsal, uma vez que o articulador designado das vogais é [dorsal]. Logo, no caso dos marcadores de efeito 0 , a vogal será antecedida por [W] ou [j], glides, portanto, derivados, que, como as vogais, apresentam [dorsal] como articulador designado, o que pode ser visto em (8) e (6); já no caso dos marcadores de efeito 1, que fazem com que o segmento inicial seja [-contínuo], a vogal será antecedida por [g].

Conforme explica Levi, VPT não conseguiria explicar estas alternâncias. Como pode ser visto em (4), /w/ possui [labial] e [dorsal] no ponto de $C$, enquanto /j/ apresenta [coronal] no ponto de $C$, os mesmos pontos apresentados pelas vogais, com a diferença de que, nas vogais, tais pontos estão sob ponto de $V$, nó ausente nos glides subjacentes. A alternância entre /w/ e [b], como mostra a autora, pode ser explicada em VPT, porque o glide possui [labial] em seu ponto de C. De maneira semelhante, a alternância entre /j/ e [f] pode ser explicada, tendo em vista que o glide apresenta [coronal] em seu ponto de C. Entretanto, não há como explicar por que o glide derivado [j] alterna com [g], em (10d). Neste contexto, deveria ser esperada uma consoante não contínua [coronal].

A partir das evidências trazidas por Levi (2004), de que (i) glides subjacentes existem e (ii) glides subjacentes se distinguem de vogais através de traços, na próxima seção discutiremos o estatuto do glide em PB. 


\section{Evidências para a existência de apenas glides deriva- dos em PB}

Não há dúvidas de que existem glides em PB. Nosso objetivo, nesta seção, é trazer evidências de que os glides e as vogais não possuem diferenças de comportamento nessa língua. A distribuição predizível entre vogais e glides que ocorre no PB é um argumento favorável à hipótese de que os glides são derivados. Em línguas em que há glide subjacente, glides são encontrados nos mesmos contextos em que vogais ocorrem, não sendo possível, portanto, uma predizibilidade na sua distribuição. Além disso, o processo de palatalização de [t] e [d] mostra que glides e vogais não são distintos em termos de traços em PB, o que é mais um argumento favorável ao glide derivado. Por fim, analisaremos um caso de alomorfia, que não traz evidências para a existência de glides subjacentes em PB.

Com relação às vogais altas subjacentes em PB, é possível estabelecer uma tendência geral de realização. Quando essas vogais estiverem no contexto interconsonantal, a realização será com uma vogal alta, como mostram os dados em (11a). A realização será com um glide quando essas vogais estiverem diante de outra vogal (11b), como mostra Simioni (2005), ou após outra vogal (11c). A realização das vogais altas pré-vocálicas é variável, mas interessa-nos, neste momento, a possibilidade de que sejam realizadas como glides. Essa é a distribuição da maior parte das vogais altas em PB, embora haja outras questões envolvidas na realização das vogais altas que as fazem fugir desse padrão. Interessa-nos, aqui, o fato de que a realização da vogal alta é, em grande medida, predizível. 
A representação subjacente dos glides e suas consequências...

Taíse Simioni

(a)

$\begin{array}{llll}\text { fita } & / \mathrm{i} / & \rightarrow & {[\mathrm{i}]} \\ \text { pimenta } & / \mathrm{i} / & \rightarrow & {[\mathrm{i}]} \\ \text { muro } & / \mathrm{u} / & \rightarrow & {[\mathrm{u}]} \\ \text { furado } & / \mathrm{u} / & \rightarrow & {[\mathrm{u}]}\end{array}$

(b)

$\begin{array}{llll}\text { afiado } & / \mathrm{i} / & \rightarrow & {[\mathrm{j}]} \\ \text { desmiolado } & / \mathrm{i} / & \rightarrow & {[\mathrm{j}]} \\ \text { ruela } & \mathrm{u} / & \rightarrow & {[\mathrm{w}]} \\ \text { enluarada } & / \mathrm{u} / & \rightarrow & {[\mathrm{w}]}\end{array}$

(c)

$\begin{array}{llll}\text { afoito } & / \mathrm{i} / & \rightarrow & {[\mathrm{j}]} \\ \text { coitado } & / \mathrm{i} / & \rightarrow & {[\mathrm{j}]} \\ \text { mau } & / \mathrm{u} / & \rightarrow & {[\mathrm{w}]} \\ \text { austero } & / \mathrm{u} / & \rightarrow & {[\mathrm{w}]}\end{array}$

Além da parcial predizibilidade de realização das vogais altas, podemos trazer como evidência para a ausência de glides subjacentes em PB um processo cujo gatilho é uma vogal alta. Trata-se da palatalização de [t] e [d] diante de uma vogal alta, como em /tigre/ $\rightarrow$ ['tyi.gri] ou /divida/ $\rightarrow$ ['dzi.vi.da]. Esse processo ocorre também diante do glide [j], como observamos em (12).

(12)

$\begin{array}{lll}\text { /patio/ } & \rightarrow & \text { ['pa.tjju] } \\ \text { /fatiado/ } & \rightarrow & \text { [fa.'tjja.du] } \\ \text { /asedio/ } & \rightarrow & \text { [a.'s } \varepsilon . d 3 j u] \\ \text { /adiado/ } & \rightarrow & \text { [a.'dzja.du] }\end{array}$


A representação subjacente dos glides e suas consequências...

Vejamos o que seria esperado se houvesse glides subjacentes em PB e se as palavras em (12), por exemplo, possuíssem estes segmentos no lugar da vogal alta subjacente. Como a palatalização é um processo que envolve o nível dos traços, a marcação lexical e o uso do traço [consonantal] não teriam muito a nos dizer em função de que a posição silábica e o traço [consonantal] não parecem ser relevantes para a palatalização. Levando em consideração essas duas propostas de representação para o glide subjacente, a palatalização que ocorre em PB não é argumento favorável à hipótese de que os glides nessa língua são derivados. Entretanto, como mostra Levi (2004), essas representações são as menos interessantes, visto que não são capazes de explicar grande parte dos processos discutidos pela autora, como mencionamos anteriormente, uma vez que tais processos fazem menção ao nível dos traços e a traços diferentes do [consonantal].

Por VPT, diferentemente, podemos analisar a palatalização como um processo em que o ponto de $\mathrm{V}$ da vogal /i/, que é [coronal, -anterior], espraia-se para a consoante anterior, criando um segmento complexo (a palatalização pode ser interpretada como um fenômeno que envolve o traço [+alto]; esta possibilidade, entretanto, não está sendo considerada aqui). Como, nessa representação, o glide subjacente possui os mesmos traços, porém sob o ponto de $C$, a palatalização ocorreria também. Portanto, VPT não nos ajuda a distinguir glides subjacentes de vogais, considerando o processo de palatalização que ocorre em PB. Diferentemente, segundo RAT, a palatalização poderia ser analisada como um processo de espraiamento do traço [-anterior], contrastivo para a vogal /i/, mas não contrastivo para o glide /j/. Para tanto, teríamos de admitir que o processo de palatalização é sensível a traços contrastivos. Se isso for verdadeiro, então temos um argumento favorável à hipótese de que os glides em (12) são derivados.

Por fim, analisaremos um processo de alomorfia que ocorre em $\mathrm{PB}$, comparando-o com um processo de alomorfia que ocorre na língua karuk. Segundo Levi (2004), nesta língua, o sufixo de futuro possui dois alomorfes: [=awif] e [=e:f] (a autora utiliza o sím- 
A representação subjacente dos glides e suas consequências...

Taíse Simioni

bolo ' $=$ ' para indicar "afixos sintáticos"). O primeiro é anexado a verbos que terminam em vogais e glides derivados, como mostram os dados em (13). Conforme a autora, nessas formas, uma das vogais em fronteira morfológica é apagada e há um processo de coalescência entre as outras duas.

(a)

Verbos que terminam em $\mathrm{V}$

[ni?áho:=wif] 'eu caminharei'

(cf. [ni?áho:] 'eu caminho')

(b)

$$
\begin{aligned}
& \text { Verbos que terminam em } / \mathrm{u} / \rightarrow[\mathrm{w}] \\
& \text { /u-ihruu-awis/ } \rightarrow \text { [?uhrô:=wif] 'ele usará' } \\
& \text { (cf. [?úhruw] 'ele usa') } \\
& \text { (cf. [ihruw] 'usar') } \\
& \text { /u-ikjau-awis/ } \rightarrow \text { [?ukjâ:wif] 'ele fará' } \\
& \text { (cf. [?úkjaw] 'ele faz') }
\end{aligned}
$$

(c)

Verbos que terminam em $/ \mathrm{i} / \rightarrow$ [j]

/ni-axai-awis/ $\rightarrow$ [ni?áxa:wif] ' 'eu pegarei'

(cf. [?axaj-tfákif(rih)] 'pegar')

(cf. [ni?áxaj] 'eu pego')

Fonte: Levi $(2004$, p. 167)

O sufixo [=e:[], por sua vez, é anexado a verbos que terminam em consoantes e glides subjacentes, como pode ser observado em (14). É necessário que se façam algumas observações neste momento. Segundo Levi, glides subjacentes, ao contrário de vogais, realizam-se como segmentos nasais diante de um morfema iniciado por consoante, como vimos na seção 2.2. Além disso, glides subjacentes não são apagados diante de um sufixo iniciado por vogal, ao contrário do que acontece com as vogais e os glides 
A representação subjacente dos glides e suas consequências...

Taíse Simioni

derivados (o apagamento das vogais e dos glides derivados neste contexto pôde ser observado nos dados em (13)). Por fim, é preciso mencionar que, conforme Levi, apenas o glide /w/ é subjacente em karuk; o glide [j] é sempre derivado.

(a)

Verbos que terminam em $\mathrm{C}$

$\begin{array}{ll}\text { [?u-Pif=e:[] } & \text { '(isto) crescerá' } \\ \text { [kuniftúk=e:[] } & \text { 'eles picarão' } \\ \text { [?uwaxráh=e:]] } & \text { '(isto) secará' }\end{array}$

(b)

Verbos que terminam em /w/

[?u-Páw=e:J] 'ele comerá'
(cf. [?am-kira] 'lugar para comer=mesa')
(cf. [?áw-iruw] 'comer muito')
(cf. [?u-Paw] 'ele come)
[nup-ápiw=e:f] $\quad$ 'ele procurará'
(cf. [?ápiw] 'procurar')
(cf. [?apim-tih] 'estar procurando')

Fonte: Levi (2004, p. 166)

Em PB, há alomorfia no sufixo de diminutivo (BISOL, 2010, 2011). São dois os alomorfes: -inho(a) e -zinho(a). Basicamente, o primeiro se anexa a palavras terminadas em vogal não acentuada, como pode ser visto em (15). Neste caso, a vogal da raiz é apagada. Já -zinho se junta a palavras terminadas em consoante, como os dados em (16) mostram. No caso de palavras como "refém" e "hífen", em (16), para afirmar que elas terminam em consoante, estamos nos apoiando na análise de Bisol (1998), segundo a qual, subjacentemente, há uma sequência vogal + consoante nasal ao final de palavras como essas. Não discutiremos, aqui, a representação fonológica dessa consoante nasal. $\mathrm{Na}$ análise da alomorfia 
A representação subjacente dos glides e suas consequências...

Taíse Simioni

de diminutivo, estão sendo desconsideradas, aqui, três situações: raízes terminadas em vogais acentuadas (café $\rightarrow$ cafezinho); raízes com acento proparoxítono (árvore $\rightarrow$ arvorezinha) e variação na seleção dos alomorfes (baldinho baldezinho). Acreditamos que essas situações não invalidam o que será afirmado adiante.

(15)

$$
\begin{array}{lll}
\text { casa }+ \text { inha } & \rightarrow & \text { casinha } \\
\text { pato }+ \text { inho } & \rightarrow & \text { patinho } \\
\text { quente }+ \text { inho } & \rightarrow & \text { quentinho }
\end{array}
$$

$$
\begin{aligned}
& \text { colar } \rightarrow \quad \text { colarzinho } \\
& \text { pôquer } \rightarrow \text { poquerzinho } \\
& \text { refém } \rightarrow \quad \text { refenzinho } \\
& \text { hífen } \rightarrow \quad \text { hifenzinho }
\end{aligned}
$$

Precisamos, agora, observar o sufixo selecionado por palavras terminadas em glide. Como pode ser visto em (17), tais palavras selecionam -zinho como sufixo, o que demonstra que esses glides estão se comportando como as consoantes de (16) e diferentemente das vogais de (15). Isto poderia sugerir que tais glides são subjacentes, uma vez que seu comportamento se iguala ao das consoantes, como acontece em karuk. Entretanto, em termos de traços, pensando em uma análise por RAT, não há nada que faça com que as consoantes de (16) formem uma classe natural com os glides subjacentes. O que há em comum entre eles é o traço [+soante], exigência feita às codas do português, mas as vogais também possuem esse traço. O que está em jogo aqui é a estrutura silábica das palavras: palavras terminadas em coda selecionarão o sufixo -zinho. Não há, portanto, evidências da presença de um glide subjacente neste processo de alomorfia. Há, por outro lado, uma evidência para a posição de coda ocupada pelo glide pós-vocálico na estrutura silábica. 
A representação subjacente dos glides e suas consequências...

$\begin{array}{lll}\text { pai } & \rightarrow & \text { paizinho } \\ \text { pônei } & \rightarrow & \text { poneizinho } \\ \text { chapéu } & \rightarrow & \text { chapeuzinho }\end{array}$

Não foram encontradas evidências, portanto, de que em PB existam glides subjacentes (interpretamos, seguindo Bisol (1989, 1999), as sequências [kw] e [gw], como em quarto e guaraná, por exemplo, como consoantes complexas, em que a articulação primária é dorsal, e a secundária, labial). Na sequência, a tipologia proposta por Levi (2004) será apresentada. Essa tipologia está baseada na ausência ou presença de glides subjacentes e derivados nas línguas do mundo.

\section{A tipologia proposta por Levi (2004)}

Segundo Levi (2004), a "dualidade" no comportamento dos glides é um dos fatores responsáveis pelas mais variadas interpretações na literatura a respeito desse segmento. Glides apresentam características tanto de vogais quanto de consoantes. Para a autora, como vimos anteriormente, existem glides subjacentes que se comportam como consoantes. Entretanto, a existência de glides subjacentes não implica, necessariamente, na ocorrência de diferenças fonéticas entre glides subjacentes e glides derivados. Levi (2004) afirma que "esses dois tipos de glides têm diferenças estruturais subjacentemente, que também são mantidas na superfície" (LEVI, 2004, p. 2). Levi (2008) discute essa questão mais extensamente. Levando em consideração que, segundo a autora mostra, algumas línguas podem apresentar diferenças fonéticas entre um glide derivado e um subjacente e outras podem não apresentar tal distinção, Levi (2008) conclui que "diferenças fonológicas devem basear-se no comportamento fonológico, não no fonético" (LEVI, 2008, p. 1977). Embora defenda a existência de glides subjacentes, Levi (2004) destaca que isso não significa que todas as línguas 
A representação subjacente dos glides e suas consequências...

Taíse Simioni

em que há glides derivados apresentam glides subjacentes. Como acabamos de ver, este é o caso do PB, ou seja, uma língua em que há, inquestionavelmente, glides derivados, mas na qual não há evidências para a existência de glides subjacentes.

Em sua análise, Levi (2004) se concentra na alternância entre vogais altas e glides ([i] [j] ou $[\mathrm{u}] \sim[\mathrm{W}]$ ), embora a autora não negue a alternância entre vogais médias e glides e a existência de glides médios ([o] e [e]). Considerando que vogais altas podem se realizar como vogais altas, como glides altos ou como ambos, e que glides altos, da mesma forma, podem se realizar como vogais altas, como glides altos ou como ambos, Levi (2004) propõe a tipologia de mapeamento apresentada em (18), em que $V$ representa uma vogal alta, e G, um glide.

(18)

\begin{tabular}{|c|c|c|c|c|c|c|c|}
\hline I & II & III & IV & V & VI & VII & VIII \\
\hline $\mathrm{V} /$ & /G/ & $/ \mathrm{V} /$ & $/ \mathrm{G} /$ & $\begin{array}{cc}/ \mathrm{V} / & / \mathrm{G} / \\
\mid & \mid\end{array}$ & $/ \mathrm{V} / \mathrm{G} /$ & ${ }_{\mathrm{I} / \mathrm{V} / \mathrm{G} /}$ & $\stackrel{/ \mathrm{V} /}{\mathrm{I} / \mathrm{G} /}$ \\
\hline
\end{tabular}

[V]

[G]

[V] [G]

[V] $[\mathrm{G}]$

[V] $[\mathrm{G}]$

[V] [G]

[V] [G]

[V] [G]

Fonte: Levi (2004, p. 9)

Segundo Levi, entre as línguas em que há glide subjacente (tipos II, IV, V, VI, VII e VIII), o tipo mais comum é o V, aquele em que uma vogal é sempre realizada como uma vogal e um glide é sempre realizado como um glide. Para a autora, esta predominância poderia ser explicada por uma maior estabilidade desse sistema, o que geraria menos confusão entre os segmentos subjacentes.

Levi não encontrou em sua análise línguas que representassem os tipos IV e VII. Uma possível explicação para a não-ocorrência do tipo IV, segundo a autora, é o fato de que ele reúne duas condições bastante raras nas línguas do mundo: a inexistência de vogais altas subjacentes e a ocorrência de glides subjacentes. A ausência do tipo VII é explicada em função de haver uma outra possibilidade de análise para línguas em que alguns segmentos 
A representação subjacente dos glides e suas consequências...

apresentam uma alternância entre vogal e glide na superfície, enquanto outros segmentos sempre se realizam como vogais. Tal alternativa de análise será mostrada logo adiante, quando for apresentada a discussão de Levi sobre a diferença entre línguas "normais" e línguas "vocálicas".

Interessa-nos discutir a que tipo pertence o PB. Como vimos, não há evidências de que os glides do PB sejam subjacentes, o que nos leva a assumir que o PB pertence ao tipo III, repetido em (19), ou seja, uma língua em que não há glides subjacentes e na qual a vogal alta pode ser realizada como glide ou como vogal alta, dependendo do contexto em que ocorre.

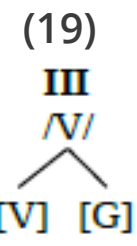

Fonte: Levi (2004, p. 9)

Para finalizar esta seção, mencionaremos a distinção que Levi (2004) faz entre línguas "normais" e línguas "vocálicas". Levi (2004) dedica um capítulo de sua tese às línguas que não possuem glides subjacentes. Segundo a autora, essas línguas podem ser divididas em "normais" e "vocálicas". Ao primeiro tipo pertencem as línguas em que a distribuição entre glides e vogais é plenamente predizível. Nessas línguas não aparecem contrastes entre vogais e glides. Ao tipo "vocálico" pertencem as línguas em que a distribuição entre glides e vogais é predizível, mas há palavras nas quais são encontradas vogais em contextos em que glides seriam esperados. Para Levi, tais palavras podem ser analisadas como possuindo uma vogal marcada para ser núcleo de sílaba. Nessas línguas, então, há vogais marcadas que nunca alternam com glide e vogais que alternam entre glides e vogais, conforme (20), em que "/i=N/" representa uma vogal alta marcada para ser núcleo. Essa situação, conforme Levi, aproxima-se do que seria o tipo VII de língua (21). 
A representação subjacente dos glides e suas consequências...

Taíse Simioni

(20)

$/ \mathrm{i}=\mathrm{N} / / \mathrm{i} /$

I 1

[i] [j]

(21)

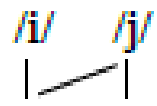

[i] [j]

A autora explica que, para distinguir as representações em (20) e (21), seria necessária a constatação de processos que pudessem distinguir os traços dos glides subjacentes em relação aos traços da vogal subjacente. Se os segmentos que sempre se realizam como vogal são atingidos pelos mesmos processos que afetam os segmentos alternantes, então há evidências para (20). Por outro lado, se os segmentos que sempre se realizam como vogal são afetados por processos que não atingem os segmentos alternantes, há evidências para (21). Conforme mencionamos acima, Levi não encontrou línguas que representassem o tipo VII.

O espanhol, segundo Levi (2004), é um exemplo de língua "vocálica", como atestariam os pares análogos em (22). Os exemplos utilizados por Levi são de Harris e Kaisse (1999, p. 123) e pertencem ao castelhano standard. $\mathrm{O}<h>$ representa a letra $h$ que não tem reflexos na fala.

(22)

$\begin{array}{llll}\text { Z[u.'a]vo } & \text { 'nome próprio' } & \mathrm{s}[\text { 'wa]ve } & \text { 'suave' } \\ \mathrm{s}[\mathrm{u} . \text { 'e]co } & \text { 'sueco' } & \mathrm{z}[\text { 'we]co } & \text { 'sapato de madeira' } \\ \mathrm{h}>\text { [u.'i]da } & \text { 'fuga' } & \mathrm{c}[\text { 'wi]da } & \text { 'ele/ela cuida' } \\ <\mathrm{h}>\text { [u.i]dízo } & \text { 'fugidio' } & \mathrm{c}[\text { wi]dádo } & \text { 'cuidado' }\end{array}$

Fonte: Harris e Kaisse (1999, p. 123) 
A representação subjacente dos glides e suas consequências...

Taíse Simioni

Harris e Kaisse (1999) e Roca (1997) tratam esses casos, em que há uma vogal onde um glide seria esperado, como palavras em que a vogal alta é marcada para ser núcleo de sílaba. Harris e Kaisse, entretanto, mencionam um processo de "desnuclearização", pelo qual vogais altas deixam de ser núcleos e passam a ocupar as margens de uma sílaba. Segundo Harris e Kaisse (1999), a partir desse processo, "contrastes como $<\mathrm{h}>[\mathrm{u}$. 'i]da vs. c[' wi]da [...] neutralizam-se como $\langle\mathrm{h}\rangle$ ['wi]da e c['wi]da sob condições de velocidade da fala e registro, que variam entre os dialetos" (HARRIS; KAISSE, 1999, p. 139).

Levi destaca que apenas a presença de pares como os de (22) não é suficiente para que se admita que uma determinada língua possui glides subjacentes. Para isso, é necessário, como vimos acima, a existência, em tal língua, de processos que façam referência ao nível dos traços e que distingam vogal e glide subjacente por atingirem os dois segmentos de maneira diferente.

O PB não possui pares análogos como os observados acima ou como os do italiano, apresentados em (23), e do russo, apresentados em (24). Isto, por enquanto, poderia nos levar a concluir que o PB é uma língua "normal". Entretanto, um quadro mais complexo de realização das vogais altas se apresenta em PB, como discutimos em Simioni (2011), quando foi possível observar que, em PB, para que uma análise dê conta da silabificação das vogais altas, a atribuição do acento tem de ser considerada, uma vez que há interação entre silabificação e atribuição de acento.

(23)

$\begin{array}{llllll}\text { fiala } & \text { ['fja:.la] } & \text { 'frasco' } & \text { viale } & \text { [vi.'a:.le] } & \text { 'avenida' } \\ \text { diavolo } & \text { ['dja.vo.lo] } & \text { 'diabo' } & \text { dialogo } & \text { [di.'a.lo.go] } & \text { 'diálogo' } \\ \text { piano } & \text { ['pja.no] } & \text { 'plano' } & \text { piano } & \text { [pi.'a:.no] } & \text { 'de Pio' } \\ \text { piovere } & \text { ['pjo.ve.re] } & \text { 'chover' } & \text { pioniere } & \text { [pi.o.'njE:.re] 'pioneiro' }\end{array}$

Fonte: Krämer (2006, p. 6) 
A representação subjacente dos glides e suas consequências...

$\begin{array}{ll}\text { i'on } & \text { 'íon' } \\ \text { i'udə } & \text { 'Judas' } \\ \text { pi'astr } & \text { 'piastra' } \\ \text { di'agnəs } & \text { 'diagnóstico' } \\ \text { xri'Isti'anstvə } & \text { 'cristianismo' }\end{array}$

(24)

Fonte: Padgett (2008, p. 1942)

\section{Conclusão}

A exposição de como Levi (2004) defende a existência de glides subjacentes nos permitiu observar mais acuradamente os glides do PB. Segundo a autora, será possível constatar a presença de glides subjacentes em uma língua se ela apresentar fenômenos em que vogais e glides se comportam de maneira diferenciada em relação aos traços envolvidos.

Uma vez que não foi possível localizar em PB tais fenômenos, restou a conclusão de que não existem glides subjacentes em PB; os glides que encontramos nessa língua são todos derivados, como pudemos perceber a partir (i) da predizibilidade parcial da realização das vogais altas, (ii) da análise do processo de palatalização de $t$ e $d$ e (iii) da discussão sobre a alomorfia do morfema de diminutivo.

Com relação à tipologia das línguas propostas por Levi (2004), a conclusão de que não há glides subjacentes em PB nos possibilitou incluir essa língua entre aquelas que apresentam apenas vogais na subjacência, mas nas quais tais vogais podem se realizar como glides ou como vogais. Dentro dessa tipologia, enquadramos o PB como uma língua "normal", no sentido de que não são encontradas vogais onde seria esperado um glide. 
A representação subjacente dos glides e suas consequências...

Taíse Simioni

\section{Referências}

BISOL, L. O ditongo na Perspectiva dA fonologia ATUAL, D.E.L.T.A., v. 5, N. 2, P. 185-224, 1989.

BISOL, L. Ditongos derivados, D.E.L.T.A., v. 10, N. Especial, P. 123-140, 1994.

BISOL, L. A nasalidade, um velho tema. D.E.L.T.A. [online], v. 14, N. EspeCIAL, 1998.

BiSOL, L. A sílaba e seus Constituintes. In: NEVES, M. H. de M. GramátiCA do português falado (V. VII). 2. ed. SÃo Paulo: Humanitas; Campinas: EDITORA DA UNICAMP, 1999. P. 701-742.

BISOL, L. O DIMINUTivo e suAS DeMANDAS, D.E.L.T.A., v. 26, N. 1, P. 59-85, 2010.

BISOL, L. O diminutivo e SUAS DEMANDAS, UMA VerSÃo REVISITADA, ReVEL, ED. ESPECIAL, N. 5, P. 80-98, 2011.

CALABRESE, A. A CONSTRAINT-BASED THEORY OF PHONOLOGICAL MARKEDNESS AND SIMPLIFICATION PROCEDURES, LINGUISTIC INQUIRY, V. 26, N. 3, P. 373-463, 1995.

CLEMENTS, G. N. Place of articulation in consonants and vowels: a unified theory, Working papers of the Cornell Phonetics Laboratory, n. 5, P. 77-123, 1991.

ClementS, G. N.; HUME, E. The Internal Organization of Speech Sounds. In: GOlDSMith, J. The Handbook of Phonological Theory. OXFORD: BASIL BLACKWELL, 1995. P. 245-306.

CLEMENTS, G. N.; KEYSER, S. J. CV PHonology: a generative theory of the syllable. Cambridge, MA: The MIT Press, 1983.

HALL, Tracy Alan. Underlying and derived glides in Middle High German, GLOSSA: A JOURNAL OF GENERAL LINGUISTICS, v. 2, N. 1, P. 1-31, 2017.

Halle, M. Feature Geometry and Feature Spreading, Linguitic Inquiry, v. 26, N.1, P. 1-46, 1995. 
A representação subjacente dos glides e suas consequências...

Taíse Simioni

HALLE, M et al. A. On feature spreading AND the Representation of place of ARTICULATION, LINGUISTIC INQUIRY, v. 31, N. 3, P. 387-444, 2000.

HARRIS, J. W.; KAISSE, E. M. PALATAL VOWELS, GLIDES AND OBSTRUENTS IN Argentinian Spanish, Phonology, N. 16, P. 117-190, 1999.

HAYES, B. Compensatory lengthening IN MORAIC PHONOLOGY, Linguistic INQUIRY, v. 20, N. 2, P. 253-306, 1989.

HUANG, Hui-Chuan J. Phonological patterning of PReVocalic GLides in Squliq Atayal, Language and Linguistics, v. 15, N. 6, P. 801-824, 2014. HUME, E. Representing the duAlity of GLIDES. IN: TSOULAS, G.; NASH, L. Actes du congrès: langues e grammaire. Paris: Department of Linguistics, UNIVERSITÉ PARIS 8, 1995.

HUME, E.; ODDEN, D. Reconsidering [consonantal], Phonology, n. 13, P. 345-376, 1996.

hyMAN, L. M. A Theory of Phonological Weight. Stanford: CSLI PubliCATIONS, 2003.

JAGGERS, ZaCcary SCOTt. EVIDENCE AND CHARACTERIZATION OF A GLIDE-VOWel distinction in American English, Laboratory Phonology, v. 9, n. 1, p. 1-27, 2018.

KRÄMER, M. What Kind of species are Italian gLides? TalK PRESENTEd at the Workshop "Phonological Bases of Phonological Features", 28-29 SepTEMBER 2006, CASTL, TROMSø.

LEVI, S. V. The RePresentation OF UNDERLYING GLIDES: A CROSS-LINGUISTIC Study. 2004. 282 f. Tese (Doutorado em Filosofia) - Departamento de LINGUística, UniVERSITY OF WASHINGTON, WASHINGTON, 2004.

LEVI, S. V. Phonemic Vs. Derived Glides, Lingua, v. 118, P. 1956-1978, 2008.

LEVI, S. V. A metrical theOry of Syllabicity. 1985. 338 f. TeSE (Doutorado em Filosofia) - Departamento de Linguística e Filosofia, Mit. Massachusetts, 1985.

LOPEZ, B. S. The sound pattern of Brazilian Portuguese (Carocian Dialect). 1979. 265 F. TeSE (Doutorado), University of CAlifornia. Los 
A representação subjacente dos glides e suas consequências...

Taíse Simioni

ANGELES, 1979.

NEVINS, A.; CHITORAN, I. Phonological Representations AND the VARIABLE PATTERNING OF GLIDES, LINGUA, v. 118, P. 1979-1997, 2008.

PADGETT, J. Glides, Vowels, and Features, Lingua, v. 118, p. 1937-1955, 2008.

ROCA, I. There are no "Glides", at least in Spanish: an optimality account, Probus, v. 9, P. 233-265, 1997.

ROSENTHALL, S. VoWeL/GLIDE ALTERNATIONS IN A theORY OF CONSTRAINT interaction. 1994. 243 F. TeSe (Doutorado), University of Massachusetts-AMHERST, 1994.

SIMIONI, T. A ALTERNÂNCIA ENTRE DitONGO CRESCENT E HIATO EM PORTUGUÊS: uma ANÁlise otimalista. 2005. 156f. Dissertação (Mestrado em Teoria e Análise Linguística) - Instituto de Letras, Universidade Federal do RıO Grande do Sul, Porto Alegre, 2005.

SIMIONI, T. UMA ANÁLISE DOS VOCOIDES ALTOS EM PORTUGUÊS BRASILEIRO: RELAÇÕES ENTRE SILABIFICAÇÃO E ATRIBUIÇÃO DO ACENTO. 2011. 147f. TESE (Doutorado em TeOrIa e AnÁlise Linguística) - Instituto de Letras, Universidade Federal do Rio Grande do Sul, Porto Alegre, 2011.

WAKSLER, R. A FORMAL ACCOUNT OF GLIDE/VOWEL ATERNATION IN PROSODIC theory. 1990. 226 f. Tese (Doutorado em Filosofia) - Departamento de Linguística, Harvard University, Cambridge, 1990.

ZeC, D. The Syllable. In: de LACY, P. The Cambridge Handbook of Phonology. Cambridge: Cambridge University Press, 2007. P. 161-194. 